\title{
PROCESOS DE FLEXIBILIZACIÓN DE LAS RELACIONES LABORALES Y LA NEGOCIACIÓN COLECTIVA EN ARGENTINA. CONTINUIDADES Y RUPTURAS DESDE LA IMPLEMENTACIÓN DEL PROGRAMA NEOLIBERAL ${ }^{1}$
}

\section{Processes of flexibilization of labour relations and collective negotiation in Argentina. Continuities and ruptures since the implementation of the neoliberal agenda}

\author{
Ana Drolas ${ }^{2}$ \\ Marcelo Delfini ${ }^{3}$ \\ Juan Montes Cató ${ }^{4}$ \\ Universidad de Buenos Aires, Buenos Aires, Argentina.
}

Recibido: 30 de octubre de 2011. Aprobado: 27 de febrero de 2012.

\section{RESUMEN}

Este artículo tiene como objetivo establecer continuidades y rupturas en las prácticas de gestión de la fuerza de trabajo vinculadas a las lógicas de flexibilidad laboral durante los periodos 1990-2002 y 2003-2011. Para ello tomamos como emergente empírico al sector automotriz argentino en esas etapas, a partir del análisis comparativo de las negociaciones colectivas de trabajo celebradas entre las diferentes firmas del sector y sus entidades sindicales. En los primeros apartados se precisa el corpus conceptual utilizado para la comprensión de la temática, para luego desarrollar las características del caso como ejemplo paradigmático en tanto sector productivo emblemático de la estructura productiva argentina.

1 Este artículo es el resultado de un proyecto de investigación UBACyT titulado "La gestión de la fuerza de trabajo en las empresas multinacionales y su impacto sobre las relaciones laborales en la firma. Entre la convergencia y la divergencia de estrategias", N²0020100200123-Programación Científica 2011-2013.

2 Dra. en Ciencias Sociales de la Universidad de Buenos Aires, DEA en Sociología de la Universidad Complutense de Madrid, Investigadora Asistente del CONICET. Correo electrónico: mdrolas@ceil-conicet.gob.ar

3 Dr. en Ciencias Sociales de la Universidad de Buenos Aires. Investigador asistente del CONICET/UNGS. Docente de la Facultad de Ciencias Sociales de la Universidad de Buenos Aires. Correo electrónico: mdelfini@conicet.gov.ar

4 Dr. en Ciencias Sociales de la Universidad de Buenos Aires. Investigador adjunto del CONICET. Docente de la Facultad de Ciencias Sociales de la Universidad de Buenos Aires. Correo electrónico: jmontes@ceil-conicet.gob.ar 
PALABRAS CLAVE: flexibilización laboral, relaciones laborales, negociación colectiva.

\begin{abstract}
This paper is intended to establish the continuities and ruptures of the management practices in the workforce linked to labor flexibility logics for the two periods 1990-2002 and 2003-2011. For this purpose we take as emerging empirical, the argentine automotive sector in those stages from comparative analysis of collective labor negotiations held between the various firms in the sector and the respective union bodies. In the first few sections is clarified the conceptual corpus used for the comprehension of the topic investigated to then develop the characteristics of the specific case as a paradigmatic example as iconic productive sector of the productive structure of argentina.
\end{abstract}

KEYWORDS: Labor flexibility, labor relations, collective bargaining.

\title{
I. INTRODUCCIÓN
}

En los años 90 se desarrolló un proceso de transformación de las relaciones laborales tendientes a su individualización, proceso en el que destacan la flexibilización de las condiciones de trabajo que heterogeneizaron los colectivos de trabajo y con ello desestructuraron las lógicas vinculadas al modelo fordista. A partir del año 2003, comienzan a desarrollarse transformaciones en el mercado de trabajo, en el régimen de acumulación y en el rol del Estado, que estarían trayendo cambios en los procesos de flexibilización iniciados en la década de los 90, en el marco de una revitalización del actor sindical sindical (Palomino y Trajtemberg 2007; Trajtemberg, Senén y Medwid 2008).

En este contexto, este artículo quiere establecer las continuidades y rupturas de las prácticas de gestión de la fuerza de trabajo vinculadas a las lógicas de flexibilidad laboral durante los dos periodos 1990-2002 y 2003-2011. Estas pueden evaluarse desde diferentes instancias y tomando como referencia distintas variables. En este estudio se toman los elementos vinculados a la negociación colectiva, lo cual permite acercarnos a la observación de los objetivos trazados. En este sentido, el nivel de la negociación colectiva y los contenidos que se introducen en ella son los elementos centrales que posibilitan la comparación entre los periodos. 
El punto de partida del presente análisis son los siguientes interrogantes: ¿cómo pueden analizarse las relaciones del trabajo?, ¿cómo se articularon las prácticas de flexibilización y la individualización de las relaciones laborales?, ¿qué elementos se encontraban presentes en las negociaciones de los 90 ?, ¿qué continuidades y cambios se observan en relación a la década? ${ }^{5}$

\section{EL MARCO ANALÍTICO DE LAS RELACIONES LABORALES}

La perspectiva adoptada toma como referencia una visión amplia de las relaciones laborales que incorpora las formas de gestión de la fuerza de trabajo, las lógicas que subyacen a la relación entre los actores intervinientes y a la vez el procesamiento que se da de ella en diferentes niveles, abandonando el criterio limitado que entiende las relaciones laborales solamente en torno a los aspectos normativos e institucionales. Desde esta perspectiva, Bilbao (1999) sostiene que las relaciones laborales no se limitan al momento del "uso" de la fuerza de trabajo en el espacio de la producción, sino que comprenden varios aspectos, entre los que se destacan los mecanismos de entrada y salida del puesto de trabajo, los sistemas de retribución e incentivos, los mecanismos de asignación de tareas, las formas en las que se regula la movilidad interna, las modalidades de la organización del tiempo de trabajo, los programas de formación, el reconocimiento de calificaciones y, eventualmente, los mecanismos de desarrollo de la carrera laboral. De esta forma, las relaciones laborales son concebidas de manera amplia, en la medida en que comprenden las formas de gestión de la fuerza de trabajo y de organización del trabajo entre las empresas y al interior de cada una de ellas, las regulaciones que permiten procesarlas, el nexo que vincula a los trabajadores y empleadores y el desarrollo de la relación entre los actores intervinientes.

Por otra parte, esta concepción toma en consideración los diferentes niveles en los que se inscriben. En un nivel "micro", las relaciones entabladas en el espacio de la empresa, las estrategias desplegadas en esa instancia y la dinámica adquirida por esas relaciones en el plano del proceso productivo. En este nivel se desarrollan las relaciones entre los empleadores, los trabajadores y los representantes gremiales. A su vez, las relaciones laborales refieren a la forma de gestión, a la organización del proceso productivo

5 El trabajo se ha desarrollado utilizando una metodología cualitativa, a partir de la cual se analizan Convenios Colectivos de Trabajo (CCT) de empresas pertenecientes al sector automotriz. La elección de este sector obedece a diferentes motivos: el primero, a la posibilidad de tomar diferentes firmas del sector más allá de las terminales que son exploradas habitualmente; el segundo, se vincula con que este sector aparece como un sector dinámico en la economía y de peso en el sector industrial, ya sea en términos de empleo, productividad y producto. 
y a la relación entre los empleadores y los representantes de los trabajadores. Este primer nivel es constitutivo y, en forma paralela, es el ámbito donde las prácticas de los demás niveles procesan su eficacia.

El segundo nivel "meso" se encuentra vinculado con las actividades productivas y hace referencia a los sindicatos sectoriales, cámaras empresarias y sus relaciones. En esta instancia se genera la coordinación entre las unidades productivas que integran cada actividad. Por otra parte, es el nexo entre los niveles micro y macro relacionales, lo que hace que este nivel, más allá de traducir e integrar diferentes elementos, tenga su propia dinámica de funcionamiento.

Por último, el nivel "macro" se integra en las relaciones laborales a partir de las configuraciones socioeconómicas que se desenvuelven en cada período histórico y que condicionan la forma de desarrollo de las relaciones en los otros niveles, pero además integra, a través del Estado, la diversidad de intereses que lo componen. En este nivel, las confederaciones de trabajadores, las entidades empresarias y el gobierno se constituyen en agentes claves que traducen la dinámica de las relaciones de los otros niveles (Senén González y Palomino 2006; Delfini 2010). Pero, aunque distinguibles analíticamente, estos niveles se autocondicionan y cada uno tendrá efectos sobre los otros.

Bajo esta lógica de comprensión de las relaciones laborales, la dinámica de la flexibilidad cruzará los diferentes niveles de análisis. Tomamos el concepto de flexibilidad a partir de la distinción que realiza Boyer (1987) entre flexibilidad "externa" e "interna". La primera se refiere a las posibilidades de entrada y salida de los trabajadores por medio de contratos de trabajo que posibiliten mantener los costos de la mano de obra y las relaciones entabladas entre las empresas, los sindicatos y el Estado. En cuanto a la segunda, da cuenta de las formas que adopta la fuerza de trabajo en situaciones concretas y se refiere a la polivalencia, implicación, grado de autonomía, trabajo en equipo, etc. Vinculado a ello, Boyer establece la existencia de diferentes elementos que caracterizan la flexibilidad productiva: el grado de adaptabilidad de la organización productiva (la capacidad del equipamiento de adaptarse a la demanda), la aptitud de los trabajadores para cambiar de puestos de trabajo (lo cual se integra a la flexibilidad interna y remite a las posibilidades de polivalencia de los trabajadores), las restricciones jurídicas al despido y al contrato laboral (vinculado a la flexibilidad externa, en la medida en que los empresarios se desligan de una parte importante de las responsabilidades que tenían en el pasado), la sensibilidad de los salarios (que se adaptan también a la demanda) y por último, la posibilidad de la empresa de desentenderse de costos salariales (Boyer 1987). 
Esta concepción de la flexibilidad permite una integración entre los diversos niveles, en los cuales las características asumidas por la flexibilidad, originadas en los niveles "macro" y "meso", se efectivizan en los espacios de trabajo y toman de este parte de su desarrollo.

\section{INDIVIDUALIZACIÓN DE LAS RELACIONES LABORALES DURANTE LOS 90}

Tomando como referencia lo mencionado en el apartado anterior, las relaciones laborales se desarrollan en diferentes niveles, comprenden diversas dimensiones y aspectos y, por ello, diferentes modos de análisis. En este sentido, la mirada puesta sobre la evolución de las relaciones laborales en Argentina, sobre todo aquella vinculada a los niveles "macro" y "meso" y a los cambios acontecidos durante la década del 90, permite enmarcar los procesos desarrollados en los niveles "micro" en el espacio mismo de la planta, observar su efectividad y cómo se desenvuelve su reproducción.

La evolución de las relaciones laborales en Argentina debe integrarse a los procesos de transformaciones económicas, políticas y sociales que se han sucedido desde 1940 y más fuertemente desde los 70. Más allá de los cambios acontecidos en la historia política argentina, puede establecerse la aparición de ciertas regularidades hasta el golpe de 1976, regularidades que se inscriben dentro del modelo de industrialización por sustitución de importaciones (caracterizado por una fuerte intervención estatal, el fortalecimiento del poder institucional del sindicato, la extensión de la negociación colectiva centralizada por rama de actividad y el predominio relativo, en el ámbito urbano, de relaciones laborales estables y a largo plazo). A partir de ese momento y con mayor profundidad desde los primeros años de la década del 90, se vislumbran transformaciones de envergadura en los aspectos concernientes a las relaciones entre capital y trabajo.

Precisamente, durante los 90 se sucedieron una serie de transformaciones importantes en los tres niveles de las relaciones laborales, pero la impronta de esta marcha será marcada por el nivel "macro". Así, las transformaciones socioeconómicas tuvieron efectos fuertes sobre los niveles inferiores, a partir de los cambios que se manifestaron en la correlación de fuerzas sociales, que a su vez conducen el rumbo del Estado.

Por tal motivo, el diseño de las políticas laborales y la forma de llevar adelante los procesos de transformación en ese contexto articulaban relaciones laborales tendientes a favorecer el desenvolvimiento de políticas económicas signadas por la libertad de mercado. 
Frente a la rigidez, la crisis generalizada de los 70 y la más localizada de fines de los 80 , el sector empresario (y los organismos internacionales de crédito) exigió flexibilidad en los marcos normativos y legales de las relaciones laborales como única opción frente a la caída de las tasas de productividad y de las performances de calidad y la desregulación respecto a las formas de contratación, como única manera de resolver los problemas de la falta de empleo. De este modo, durante este período se fueron desarrollando un conjunto de leyes (especialmente la 24.013) e instrumentos que, bajo las consignas de "modernización" y creación de empleo, fueron socavando los andamiajes que sostenían los derechos laborales. Se abría la posibilidad de contratar personal por tiempo determinado, se extendían los periodos de prueba, se daba la posibilidad de fraccionar el aguinaldo, se promocionaba la contratación por medio de pasantías y se reducían las cargas patronales de las empresas con el fin de hacerlas más competitivas, inclusive para aquellas que gozaban de una inserción monopólica.

Así se impusieron la flexibilidad en relación a la organización de los puestos y a la estabilidad laboral (con el debilitamiento de los contratos con duración indeterminada y la consecuente precarización del vínculo laboral y la multiplicación de las formas de disponibilidad del trabajo al capital); el aumento de la parte variable del salario en detrimento de la fija (a partir de la instalación de novedosas formas de incentivos: premios por participación, por desempeño, por productividad individual o colectiva, etc.); los cambios en las formas de organización del trabajo sin intermediar negociación con el sindicato; la externalización de funciones productivas y no productivas, etc.

Una vez frenada la inflación por medio del plan de convertibilidad, bajo cierta esfera de legitimad derivada del disciplinamiento que significó el proceso hiperinflacionario de 1989-1990, y considerando además una nueva correlación de fuerzas donde el empresariado aparecía con mayor fortaleza y mejor vinculación con el gobierno de Menem frente al creciente desprestigio de los sindicatos, se comenzaron a establecer y llevar adelante los lineamientos de la tan mentada "modernización" de las relaciones laborales, acoplada a la "modernización" del Estado y a la "modernización" empresarial. Los efectos de esto empezaron a hacerse sentir paulatinamente a lo largo de la década de 1990.

De esta manera se pone de manifiesto una tendencia a dotar de un carácter más económico que jurídico a las relaciones laborales, con la revisión de su herramental otrora protectorio. Esta desreglamentación supuso la remoción de obstáculos que distorsionaban el mercado de trabajo, por un lado (por las dificultades para despedir y el necesario pago de indemnización), y la regulación más "libre" de los contratos y del uso de la fuerza de trabajo, por el otro, junto con el desplazamiento (especialmente en las empresas privatizadas) de la 
negociación colectiva a niveles micro, esto es, a nivel de empresa, situación otrora impensable desde el punto de vista del sindicato.

En cuanto a los contenidos de la negociación colectiva durante los 90, estos se encontraban vinculados a los elementos de flexibilidad, tanto interna como externa, exigidos por el sector empresario. Al respecto, los CCT y acuerdos celebrados entre empresarios y sindicatos incorporaban cláusulas que, partiendo de la "filosofía de la empresa", establecían las formas organizacionales por medio de equipos de trabajo, la polivalencia, la capacitación, la participación y la autonomía. En tanto, los mecanismos de flexibilidad externa se manifiestan por medio de las modalidades de contratación, el contrato por tiempo determinado y, en carácter de prueba, las posibilidades de fraccionamiento de las vacaciones y la disposición del tiempo de trabajo del asalariado por medio de la anualización de la jornada de trabajo. Precisamente, y de acuerdo con estudios realizados en diferentes sectores (Novick 1998; Montes Cató, Drolas y Picchetti 2005; Figari 2000), parecen privilegiarse aquellos aspectos vinculados a la flexibilidad externa por sobre aquellos que se encuentran dentro de los mecanismos definidos como flexibilidad interna.

Esto lo que hizo fue profundizar la individualización de las relaciones laborales y allanar el camino para la legitimación de una forma de gestión particular de la fuerza de trabajo, que Danièle Linhart (2002) llamó feudalización de la subjetividad, que integra al trabajador al espacio de trabajo en tanto individuo moral. Pero también tuvo fuerte impacto en la negociación colectiva en tanto figura legal de protección del trabajo y del trabajador, propiciando una fuerte heterogeneización de los colectivos laborales anclados en la actividad. Tal vez el paradigma de este proceso lo constituyó la ley $\mathrm{N}^{\circ} 25.250$ del año 2000, conocida como "Ley Banelco", que determinaba las modificaciones de los CCT ultraactivos ${ }^{6}$ vigentes desde la ronda negocial de 1975 y 1988, mientras otorgaba la posibilidad de establecer el predominio de un CCT de menor nivel sobre los de mayor nivel.

Lo que está en el fondo de todo este andamiaje es la búsqueda del abandono de viejas solidaridades tejidas en el marco del espacio de trabajo; es, en suma, una batalla por la identidad de los trabajadores, por ganar sus mentes (Linhart 2002).

6 Una norma solamente rige hacia el futuro, pero puede presentarse la ultractividad, es decir, cuando un CCT pierde vigencia (por no haberse firmado uno nuevo antes del vencimiento de su vigencia) sigue produciendo efectos con posterioridad a su caducidad o extinción. 


\section{ELEMENTOS DE RECOMPOSICIÓN DE LAS RELACIONES LABORALES A PARTIR DE 2003}

Luego de la crisis de empleo más aguda de los últimos 50 años, cuyos valores máximos fueron registrados en 2002 con una desocupación abierta de 22 por ciento, desde el 2003 el mercado de trabajo comienza a dar muestras de recuperación. Paulatinamente, la desocupación fue disminuyendo hasta ubicarse por debajo de los dos dígitos, al igual que el subempleo. En esta disminución tuvo un rol clave el sector industrial, que se expandió en el período 2003-2008 al 6 por ciento creando 400.000 puestos de trabajo registrados (Español y Herrera 2010).

Por su parte, la recuperación del empleo fue acompañada por una serie de reformas en la legislación que buscaron desandar algunos de los impactos más regresivos en las normativas laborales y en la seguridad social. Siguiendo a Biaffore yBerasueta (2010) se destacan avances en: a) la relación individual de trabajo en vista de abandonar las estrategias de flexibilidad de ingreso (reducción temporaria y estableciéndose limitaciones formales tendientes a evitar el fraude laboral, rescatando institutos como la integración del mes de despido, se procede a una reglamentación específica para las agencias eventuales); b) se restablece el salario mínimo, vital y móvil dejado en desuso desde 1993. A su vez, en el anterior esquema flexibilizador del salario y sus componentes, el rubro "no remunerativo" había ido en aumento, impactando en la conformación del salario y en la seguridad social. En el 2008 estos componentes comienzan progresivamente a incorporarse en el salario en carácter de remunerativo; c) se unifica el sistema de pasantías. El nuevo sistema de pasantías educativas delimita su aplicación a estudiantes universitarios, formación profesional, mayores de 18 años, y crea dispositivos de contralor para monitorear su utilización fraudulenta ocultando bajo el rótulo de formación de relaciones salariales $^{7}$; d) se reinstala la indemnización por antigüedad frente al despido sin causa. Con la sanción de la ley 25.877 se reunifica el sistema indemnizatorio frente al despido sin causa introduciendo diferentes modificaciones: la base del cálculo será la mejor remuneración mensual, normal y habitual, el monto mínimo de la indemnización será equivalente a un mes de ese salario y el cálculo se realizará en función de la fórmula que resulta más beneficiosa para los trabajadores. A su vez, en el marco de la Ley de Emergencia Económica 25.561 se aprobó la duplicación de las indemnizaciones, factor que fue decreciendo hasta el 2007, cuando la desocupación estuvo por debajo de los dos dígitos.

$7 \quad$ No obstante hay que señalar que los avances en el salario surgen luego de una devaluación (2002) que afectó el 30 por ciento de los salarios, mientras que desde el 2008 la inflación comenzó a acelerarse. 
En el plano del actor sindical también se observan señales de recomposición. Los sindicatos comenzaron a reposicionarse como interlocutores del Estado y de la sociedad en su conjunto vía la negociación salarial (Palomino y Trajtemberg 2007). El lugar que habían perdido frente a otras organizaciones sociales, como la de los movimientos de desocupados o el de las empresas recuperadas (ambas innovaciones del campo popular de una importancia enorme), comenzaba a ser recuperado por las cúpulas sindicales.

El año 2004 marca un punto de inflexión en la recuperación de las negociaciones colectivas, duplicando el promedio de los 10 años anteriores e incrementándose un 20 por ciento con respecto al 2003. De acuerdo a los datos proporcionados por el Ministerio de Trabajo, Empleo y Seguridad Social (MTESS), la cantidad de Convenios Colectivos de Trabajo homologados pasó de 380 en el año 2003 a 1.027 en 2007, y siguió incrementándose en los años 2008 y 2009 con 1.231 y 1.331 acuerdos y convenios firmados, respectivamente (MTESS 2010). De acuerdo a los datos del Ministerio, esta revitalización se refleja en todos los niveles, es decir, tanto en la reapertura de la negociación a nivel de actividad, que prácticamente se había abandonado en la década del 90, en las negociaciones por empresa, que habían mantenido su dinamismo en esa época y también en las nuevas unidades de negociación que surgieron impulsadas por el crecimiento económico (Palomino y Trajtember 2007).

Como señalaran Montes Cató, Lenguita y Varela (2010), desde el ascenso del kirchnerismo los trabajadores organizados comandaron la conflictividad laboral, la negociación colectiva y, en términos políticos, gracias a una alianza estratégica de las cúpulas sindicales con el gobierno, balancearon el peso específico que fue adquiriendo la oposición gubernamental.

Frente a esta recomposición de la capacidad de intervención de las organizaciones sindicales, se fue extendiendo un activismo de base que potenció el desarrollo de experiencias sindicales en espacios productivos donde había sido arrasada o directamente inexistente. Este fenómeno, si bien es difícil de mensurar, puede ser caracterizado a través de ciertos indicadores que permiten aproximarnos al tema en términos cuantitativos. Por un lado, se verifica, siguiendo los estudios del Observatorio del Derecho Social (2009), una mayoría de conflictos circunscritos al ámbito de la empresa, que representan el 78 por ciento del total. En segundo lugar, se consolida un crecimiento de los reclamos promovidos por sindicatos locales o seccionales, que explican el 64 por ciento de los casos en el 2009 (frente a un 51 por ciento el año anterior). En tercer lugar, persiste una importante cantidad de conflictos (un 14 por ciento del total) llevados adelante por trabajadores donde no se consigna la presencia de representantes sindicales o se trata de colectivos 
que poseen una representación de hecho (muchas veces opuesta a la conducción de la organización sindical) construida en el desarrollo del propio conflicto. Esto último es muy importante, porque señala la existencia de conflictos encabezados por delegados "de hecho" que no cuentan con ninguna cobertura legal contra represalias patronales. En términos cualitativos, las características de esa presencia sindical en los lugares de trabajo (manifiesta tanto por sus conquistas o derrotas) demuestra que no se trata solo de representaciones vaciadas de representatividad (en el caso de delegados que solo funcionan como figuras decorativas), sino que se está frente a atisbos de recomposición sindical donde prevalece el principio de mayor participación. Ello a través de la incentivación de formas de consulta de las bases, aumento en la cantidad de activistas, consolidación de los derechos de los delegados y efectivo ejercicio. Esto aparece manifestado en diferentes sectores productivos.

En este contexto de recuperación parcial de los trabajadores frente al capital, lo que se busca en los apartados siguientes es observar si ese avance en los niveles "macro" se tradujo en avances y resignificaciones en los niveles "meso" y "micro", observándose por medio de la negociación colectiva y su contenido en el sector automotriz.

\section{LA NEGOCIACIÓN COLECTIVA EN EL SECTOR AUTOMOTRIZ}

Si bien en Argentina la negociación colectiva tuvo como eje central la actividad, en el sector automotriz, y más específicamente en las terminales, se ha privilegiado la negociación por empresa, hecho que se vio profundizado durante la década del 90 por exigencia de las firmas que ingresaban al mercado local y su vínculo con las políticas neoliberales. Los cambios operados en la industria automotriz modificaron y complejizaron el desenvolvimiento de la actividad y, a su vez, las relaciones laborales. Dentro de ellas se integraron diversos actores sindicales que en periodos anteriores aparecían como marginales a dicha actividad. Precisamente, este proceso se desarrolló en el marco de un cambio tecnológico referido tanto a la utilización de materiales diversos para la construcción de automóviles, como a la incorporación tecnológica integrada al automóvil. A su vez, los procesos de tercerización y el desprendimiento de las firmas terminales de numerosas secciones de la empresa contribuyeron a la incorporación de otros actores sindicales, en la medida que se hizo más importante la presencia de otras actividades.

Esta forma de segmentar la empresa fue muy efectivo para las firmas, en la medida en que lograron deshacerse de una parte importante de trabajadores con elevados niveles salariales y, a la vez, como elemento político heterogeneizó los colectivos laborales, 
quitándoles parte del poder que los representantes podían utilizar en el marco de esas negociaciones. En este sentido, la segmentación y tercerización significó división de los colectivos de trabajo, organizados en una sola firma.

Para establecer un panorama general de los actores sindicales intervinientes, debe señalarse que en la actualidad la industria automotriz argentina se encuentra constituida por 11 empresas terminales que se dedican a ensamblar partes y piezas provistas por 500 firmas, aproximadamente, y un conjunto importante de empresas concesionarias que son las encargadas de la comercialización. Su importancia en el sector manufacturero es relativamente alta si se toma en consideración el valor agregado que produce y los puestos de trabajo que genera, además de ser una de las actividades que más ha crecido en los últimos años.

En este marco y tomando en consideración el desenvolvimiento de las relaciones laborales en Argentina, en el sector automotriz actualmente intervienen, junto con el Sindicato de Mecánicos y Afines del Transporte Automotor de la República Argentina (SMATA) y Unión Obrera Metalúrgica (UOM), otros sectores y representaciones gremiales. Tal es el caso del sector del vidrio, representado por el Sindicato de Obreros de la Industria del Vidrio y Afines (SOIVA) y el Sindicato de Empleados de la Industria del Vidrio y Afines de la República Argentina (SEIVARA); del caucho y neumático, donde también intervienen dos entidades gremiales: el Sindicato Único de Trabajadores del Neumático y Afines (SUTNA) y Sindicato de Empleados del Caucho y Afines; del sector petroquímico (Federación de Trabajadores de la Industria Química y Petroquímica) y el de pinturas (Unión del Personal de Fábricas de Pintura y Afines de la República Argentina). Existe, a su vez, representación gremial de supervisores, como en el caso de la Asociación de Supervisores de la Industria Metalúrgica de la República Argentina (ASIMRA) y la Asociación del Personal superior de Daimler Chrysler Argentina.

De estos sindicatos, SMATA, SOIVA, Federación de Trabajadores de la Industria Química y Petroquímica, y SUTNA han firmado CCT por empresas, en tanto los restantes, si bien pueden realizar acuerdos en ese nivel, no pueden ser específicamente de este tipo puesto que los CCT son a nivel de actividad.

En este contexto, se destacan por la cantidad de empresas intervinientes los que representan a los trabajadores del sector metalúrgico y, por su importancia, los que nuclean a los de firmas terminales. En el caso de las firmas metalmecánicas que intervienen en el proceso productivo, el ingreso de las grandes empresas y conglomerados autopartistas de carácter global durante la década del 90 y la posibilidad otorgada en materia regulatoria, 
hizo que algunas de estas firmas en CCT "por empresa" con el SMATA, poco frecuente durante épocas anteriores, ya que los trabajadores de los autopartistas estaban representados mayoritariamente por la UOM y se regían por CCT de actividad.

La posibilidad que otorgaba el SMATA de negociar por empresa ${ }^{8}$ hizo que se firmasen convenios entre este y algunas firmas proveedoras de las terminales, sobre todo aquellas que ingresaban con la ensambladora. De esta manera comenzaron a desarrollarse nuevas formas de articulación negocial que partían de la firma de convenios con la empresa terminal, y luego se firmaba un convenio con características parecidas o similares con las proveedoras.

A partir de la normalización de la negociación colectiva, entre 1989 y 2003 se firmaron 673 CCT por empresa, lo cual promedia un total de 45 CCT anuales. En tanto, en el período 2004-2010 el total de CCT asciende a 575, con un promedio anual mucho más elevado que en el período anterior (75 CCT por año), representando el 69 por ciento del total de CCT homologados en este período (31 por ciento se han firmado a nivel actividad). En los dos periodos analizados los CCT del sector representan el 6 por ciento del total de CCT firmados por empresa, ${ }^{9}$ los CCT homologados por las empresas no terminales representan el 78 por ciento del conjunto firmado en el sector.

\section{Cuadro $\mathrm{N}^{\circ} 1$}

CCT por empresa en la trama automotriz firmados desde 1989

\begin{tabular}{|c|c|c|c|c|c|}
\hline Año & $\begin{array}{c}\text { CCT firmados } \\
\text { por terminales }\end{array}$ & $\begin{array}{c}\text { CCT firmados } \\
\text { en el resto del } \\
\text { sector }\end{array}$ & $\begin{array}{c}\text { Total de convenios } \\
\text { firmados en sector }\end{array}$ & $\begin{array}{c}\text { \% de CCT } \\
\text { por año en } \\
\text { sector }\end{array}$ & $\begin{array}{c}\text { Total } \\
\text { CCT por } \\
\text { empresa }\end{array}$ \\
\hline $1989-2003$ & 14 & 28 & 42 & 6 & 673 \\
\hline $2004-2010$ & 2 & 29 & 31 & 6 & 525 \\
\hline Total & 16 & 57 & 73 & & 1198 \\
\hline
\end{tabular}

Fuente: Elaboración propia en base a datos de la Subsecretaría de Programación Técnica y Estudios Laborales

Analizando cada uno de los periodos se observa que, en el primero, los CCT firmados por las empresas no terminales representaban el 66 por ciento, mientras que en el segundo 93 por ciento, siendo, por tanto, menos relevante la firma de CCT en las

\footnotetext{
8 Debe aclararse, sin embargo, que SMATA no solo firma CCT por empresa, sino que también tiene CCT de actividad, como son los casos de las concesionarias ACARA y Talleres FAATRA.

9 La firma de CCT abarca tanto la firma de nuevos CCT como las renovaciones que se pudieran llevar adelante.
} 
empresas terminales. Por otra parte, se pasa de un promedio de 3 CCT anuales a 4 en el último período. Tanto en términos generales como sectoriales, puede marcarse cierta continuidad en torno a los procesos de descentralización de la negociación colectiva entre los periodos analizados, tomando como referencia la lógica de la firma de los CCT. En este sentido, como fue postulado más arriba, sigue siendo predominante la empresa como espacio de negociación, manteniéndose las lógicas de los 90. Si se observa una cierta continuidad en los términos cuantitativos de la individualización de las relaciones laborales en relación con los procesos de descentralización de la negociación colectiva, quedaría por establecer en qué medida los contenidos de la negociación muestran esta misma continuidad.

\section{CONTINUIDADES EN LAS PRÁCTICAS FLEXIBLES VISTA DESDE LOS CCT}

La revisión de los contenidos de los CCT permite acercarse al análisis de la transformación de la fuerza de trabajo en trabajo efectivo, las formas que asume su gerenciamiento y dar cuenta del sentido que adopta la organización del trabajo y los rasgos de la flexibilidad interna y externa, en la medida que estos expresan las dinámicas empresarias negociadas con los sindicatos.

Durante la década del 90 se observa una nueva lógica en lo referente al contenido de la negociación, que se inaugura en el sector automotriz con la firma del CCT que realiza SMATA con GM. Su particularidad radicaba en que se firmó antes de la instalación de la empresa y por lo tanto antes de la constitución del colectivo de trabajadores, poniendo de manifiesto la importancia que adquieren para las firmas las condiciones de desarrollo del proceso de producción y la forma en que se lleva adelante la transformación del trabajo en trabajo productivo, donde se imponen los criterios empresarios y se busca insertar criterios referidos a la flexibilidad en la asignación de tareas y lógicas de flexibilidad externa. Posteriormente, se han firmado de esta misma forma los CCT de Fiat y Toyota. En este sentido, el privilegio de la inversión y la generación de empleo que ello llevaba aparejado, de acuerdo al discurso empresarial, dinamizaba y posibilitaba, en un marco de escasez, la generación de condiciones para la firma de CCT a medida de las empresas, lo que les posibilitaba imponer ciertos mecanismos tendientes a favorecer y asegurar un mayor rendimiento de la fuerza de trabajo. De esta manera, las relaciones laborales y, dentro de ellas, las formas de organización del trabajo se articulan con las prédicas neoliberales, cuyo discurso intentaba confrontar las rigideces de las formas existentes a las bondades de la flexibilidad, que posibilitaría la generación de empleos y mejoras en él. Sin embargo, 
lejos de desarrollarse una reducción en los niveles de desempleo, la implementación de medidas flexibilizadoras durante los años 90 , produjo un incremento de la desocupación.

En cuanto al CCT firmado entre SMATA y la Empresa GM en 1994, allí se establecían criterios de polifuncionalidad, reducción del número de categorías laborales, capacitación, participación de los trabajadores y anualización de la jornada laboral a 2.188 horas, lo que inducía a la realización de cambios que hacían al mismo proceso de trabajo. También la empresa Toyota firmó un CCT antes de la conformación del colectivo laboral en 1996, luego revisado en 2001, que mantiene las lógicas centrales de la flexibilidad en torno a los contenidos negociados.

En las firmas de los convenios con algunos de los grandes proveedores de autopartes se pudo observar ciertas particularidades que hacen a las formas de ingreso al mercado local. Algunas de estas empresas firmaron CCT de trabajo con el SMATA durante la década del 90, tomando como base los que firmaban las terminales, sobre todo aquellas empresas que ingresaban junto con la empresa núcleo o eran parte del grupo. Pero, también aquellas que ingresaban al mercado local por medio de la compra de firmas nacionales o internacionales que producían en Argentina encontraban los CCT que regían en esas firmas, con lo cual en algunos casos se desarrollaban acuerdos posteriores. En el caso de las empresas vinculadas al SMATA se podían llegar a concretar nuevos CCT, puesto que con aquellas firmas vinculadas a la UOM se podían desarrollar acuerdos de vigencia determinada. Más allá de la representación gremial que correspondiera, se desarrolló un proceso de heterogeneización del colectivo laboral entre trabajadores que realizaban las mismas tareas e incluso, en algunos casos, entre trabajadores pertenecientes al mismo conglomerado. Ello podía derivar también en diferencias, no solo de condiciones de trabajo, sino también de salarios, fragmentando aún más el colectivo de trabajo.

Ejemplo de lo dicho anteriormente son los casos de las firmas de CCT con similares características por la pertenencia al grupo empresario, o por el ingreso de la firma núcleo y de la firma proveedora. Al respecto, se destacan los firmados por Fiat y las autopartistas Magneti Marelli, o los casos de GM y Delphi y Toyota y Yazaki. En estos casos, aunque existen diferencias en torno a los salarios, los procesos de trabajo que se describen son básicamente similares.

Por otra parte, en una de las siete empresas que actualmente conforma el conglomerado Dana rige el CCT firmado entre SMATA y ETON, mientras que en las otras los trabajadores son representados por la UOM, lo que implica que los trabajadores pertenecientes al mismo conglomerado no solo no tienen las mismas condiciones de trabajo, 
sino tampoco los mismos salarios. Al respecto, actualmente los salarios básicos por CCT que rigen en las empresas vinculadas a la UOM son un 17 por ciento inferior a los de la empresa del grupo vinculada con SMATA.

De esta forma no solo se divide la producción, sino que ello acarrea un proceso de diferenciación creciente entre los trabajadores que va más allá de la mera separación por empresas. Estas diferencias se dan en las condiciones de trabajo dentro de los mismos conglomerados y luego en las mismas empresas, factores que tienden a favorecer las dinámicas empresarias de individualización y pérdida del sustrato colectivo del trabajo.

Así, se pueden establecer en las negociaciones de la década del 90, por un lado, un proceso en el que tienden a prevalecer formas de flexibilidad externa e interna con intervención de los sindicatos y, por otro, procesos de descentralización de la negociación, donde el punto crucial es la negociación en el nivel "meso", que establece las prácticas, sus contenidos y reglas en el nivel inferior, adaptándose a los criterios impuestos en el ámbito de la empresa.

Los cambios en los niveles macro señalados podrían derivar en cambios en los niveles menores. Así, sería de esperar transformaciones importantes en el nivel micro, pasando de regímenes sustentados en las lógicas de flexibilidad a otros menos flexibles. Para ello, tomaremos algunos de los CCT firmados en el sector automotriz a partir de 2004, observando elementos tales como la jornada de trabajo, las formas de remuneración, la polivalencia, los criterios de participación, las normas de evaluación y las modalidades de contratación, todas ellas prácticas de gestión de la fuerza de trabajo introducidas durante la década neoliberal. En este sentido se analizan cuatro CCT, dos de los cuales son renovaciones de los firmados en la década del 90 (Toyota y Yazaki) y los otros dos corresponden a nuevos CCT (Master Trim y Pilkington) y además se incluyen otros a modo de ejemplo. En el caso de Toyota (190/96 y 730/05) se trata de los CCT firmados en 1996 y 2005, los de Yazaki (211/97 y 1033/09) en 1997 y 2009, en tanto que los de Pilkington (665/04) y Master Trim (704/05) se tratan de CCT firmados en 2004 y 2005, respectivamente.

En el caso de las dos primeras empresas se observan continuidades en torno a las lógicas de flexibilidad interna, marcadas por la fuerte impronta puesta en el trabajo en equipo, la polivalencia y la determinación de participación por parte de las firmas en círculos de calidad, Kaizen, etc., motivos que impactarán, en el caso de Toyota, sobre las probabilidades de incremento en las categorías para cada trabajador. 
$\mathrm{Al}$ analizar los diferentes convenios se observa una continuidad a privilegiar formas de organización del trabajo a través de células o equipos, con criterios de polifuncionalidad, mostrando características de flexibilidad interna o funcional en las formas organizativas.

Dentro de la "Filosofía de trabajo de la empresa", se destacan las características que distinguen a la forma de organización productiva y, en ella, la realización del trabajo por medio de equipos o células, lo cual aparece en cada uno de los CCT analizados en este apartado, tanto en términos comparativos entre CCT de las mismas firmas como en los firmados luego de la década del 90 . Se mantiene la forma de organización del trabajo que traslada al equipo de trabajo la responsabilidad de la producción: es la célula la responsable de lo producido y supone que cada uno de los integrantes sea responsable de sus tareas. Al respecto, el CCT 704/ 05 se refiere a la responsabilidad de los trabajadores en el marco del grupo y la responsabilidad del grupo con la empresa.

Es responsabilidad de los trabajadores contribuir ampliamente en la obtención de los objetivos del grupo, colaborando con los demás miembros [...] Cooperar con la empresa y los demás integrantes del equipo [...] El Trabajo en célula o en equipo, sistema que permite la asignación de responsabilidades a cada uno de los integrantes, donde los miembros de cada célula cooperan para lograr los objetivos del equipo, relacionados con la producción, calidad, reducción continua de costos, seguridad y eficiencia (CCT, 704/05).

Por su parte, otros CCT permiten observar la dimensión que cobra el trabajo en equipo, cuya responsabilidad se vincula a los destinos en la empresa, ya que los grupos aparecen como los ejes desde donde se puede incorporar nuevos elementos a la producción.

Organizar el trabajo de acuerdo a métodos modernos que privilegien el trabajo en grupos o células de trabajo que permitan la asignación de responsabilidades a cada uno de los miembros integrantes. (665/04).

Trabajo en célula o en equipo, sistema que permite la asignación de responsabilidades a cada uno de los integrantes, donde los miembros de cada célula cooperan para lograr los objetivos del equipo, relacionados con la producción, calidad, reducción continua de costos, seguridad y eficiencia (730/05).

El trabajo en equipo es esencial para la empresa y comprende: a. Trabajar juntos para alcanzar objetivos compartidos, b. Compromiso con el trabajo, c. Ser creativo, utilizando todos los recursos disponibles, d. Compartir abiertamente puntos de vista y opi- 
niones, e. Capacitarse constantemente sobre el trabajo propio y el de los demás integrantes del equipo, f. Apoyar a la empresa y los demás integrantes del equipo (730/05).

No obstante, la expresión más clara de las formas de delegación de responsabilidades al equipo de trabajo y a cada uno de los trabajadores, donde a la vez se suman un conjunto de tareas que antes se establecían en sectores diferentes, se manifiesta en el CCT de la empresa productora de parabrisas:

Los equipos o células de trabajo en su conjunto serán responsables por las funciones a su cargo, estableciéndose como filosofía de trabajo la polivalencia funcional, movilidad interna y la flexibilidad de tareas. a. La operación de la máquina y/o equipamiento productivo, o desarrollo de tareas complementarias bajo su responsabilidad siguiendo expresamente las indicaciones que surgen de las hojas de proceso, gamas, y toda otra documentación técnica que especifique procedimientos a cumplir [...], b. Deben cumplimentar los requerimientos del Programa de Aseguramiento de la Calidad y del Plan de Control de manera de evitar defectos que generen trabajos adicionales, pérdidas económicas a la empresa y una mala imagen ante nuestros clientes [...], c. La aplicación de las técnicas de Mantenimiento Total Productivo (T.P.M.) [...], d. La capacitación de nuevas personas que integrarán el equipo o célula de trabajo, e. El análisis de los problemas y sugerencias que surjan de la responsabilidad del grupo o célula de trabajo principalmente enumeradas en el presente artículo se realizará en reuniones organizadas dentro o fuera del horario de trabajo, en este último caso se abonarán como horas simples $(665 / 04)$.

Este CCT permite observar el conjunto de responsabilidades entregadas al trabajador. Este ya no es solo responsable de producir bajo el ritmo que impone la firma, sino que además debe hacerse cargo de otro conjunto de responsabilidades, que incluyen el deber de evitar trabajos adicionales, pérdidas económicas y una mala imagen ante el cliente. De esta forma, uno de los dispositivos vinculados con la flexibilidad interna mantiene su funcionamiento bajo el nuevo contexto "macro".

Otros de los elementos que se destacan en el marco de la filosofía de trabajo de las empresas y que ha sido uno de los elementos centrales de la flexibilidad interna, es el de la polivalencia y la posibilidad de que los trabajadores puedan ocupar diferentes posiciones, de acuerdo con los requerimientos de la producción y con la organización del trabajo determinado por la firma: 
Polivalencia funcional, por medio de la cual se podrá asignar al trabajador o a la célula a otras funciones y tareas, que permitirán el logro de una mejor y mayor productividad (730/05).

Es indispensable una estructura de producción y trabajo que privilegie la adaptabilidad laboral y la polivalencia en las funciones, como marco para lograr el mejor producto al más bajo costo para el mayor número de clientes. Polivalencia funcional, por medio de la cual se podrá asignar al trabajador o a la célula a otras funciones y tareas — de conformidad con las pautas de la legislación vigente-, que permitirán el logro de una mejor y mayor productividad (704/05).

Los empleados, conforme su nivel de conocimientos, podrán desempeñarse indistintamente en cualquiera de las siguientes actividades, como ser: tareas de producción, inspección, mantenimiento, montaje y armado, operación de máquinas, manejo de materiales, acondicionamiento de herramientas o elementos de trabajo, máquinas y otros elementos relativos a la actividad de la empresa, así como aquellas relacionadas directamente con las antes mencionadas y con los procesos de trabajo de la empresa (1.033/09).

De esta forma, se pone en evidencia el carácter polivalente que deben adoptar los trabajadores para adaptarse a los distintos requerimientos de las firmas y dentro de ellas, a los de la producción.

Estos elementos vinculados a la polivalencia se evidencian en las categorías incorporadas a los CCT, los cuales establecen la posibilidad, para la empresa, de asignar tareas y funciones de acuerdo a los requerimientos productivos. Lo anterior se manifiesta, por ejemplo, en el convenio firmado entre el SMATA y la empresa Toyota en 1996, donde se definen dos categorías-a diferencia de las 10 existentes en el convenio firmado con Autolatina en 1989-. Sin embargo, cabe señalar que en el CCT que rige en la firma japonesa desde 2005, si bien las categorías de trabajo son las mismas dos que en el CCT de 1996, se incorporan distintos niveles dentro de cada una de ellas, con lo cual se aligera la diferencia respecto de la multiplicidad de categorías mencionada en el convenio de 1989. El pasaje de una categoría a otra se relaciona con la antigüedad, pero sobre todo con las evaluaciones a los trabajadores (el único pasaje de nivel solamente por antigüedad es el primero correspondiente a la categoría de operario polivalente): “...la Empresa implementará un sistema de evaluación de desempeño y habilidades cuya finalidad será evaluar a cada trabajador en forma periódica, de modo de mejorar el desenvolvimiento objetivo del mismo y permitir evaluar sus posibilidades de promoción y desarrollo". (730/05). 
En el caso de las autopartistas, las categorías existentes en la empresa productora de butacas y paneles son tres, de las cuales la segunda y la tercera tienen dos niveles cada una, las de la firma productora de mazos para cable son cinco y las de la firma productora de parabrisas son cuatro. En esta última, los pasajes se producen por la antigüedad en la empresa y por los procesos de evaluación que allí se desarrollan, aunque incorpora la elección de los miembros de las células de trabajo.

En lo que se refiere a las otras dos empresas analizadas, los cambios de categorías se realizan a partir de la antigüedad en la empresa, sin que se desarrollen procesos evaluativos para ello. Lo que es importante señalar, en el marco de la definición de categorías, es la alta flexibilidad que otorga la existencia de solo unas pocas y su descripción como polivalentes, siendo posible otorgarle a un operario diversas tareas, en el marco de aquella polivalencia establecida en cada una de las pocas categorías existentes.

En torno a la jornada de trabajo, no se observan cambios entre los CCT firmados en los 90 y los actuales, manteniéndose las 2.133 horas anuales de trabajo con sistema de créditos y débitos al igual que en el CCT de Master Trim. En tanto, en el caso de la firma Pilkington se realiza un régimen plurisemanal de cálculo de la jornada de trabajo, pudiéndose trabajar los sábados y domingos, otorgando un franco compensatorio. Se llega a un promedio entre tres semanas de un mínimo de 40 y un máximo de 46,67 horas, en tanto que en una semana se puede llegar a un máximo de 52 horas. En este mismo orden, se establece en los CCT la fuerte dependencia de las firmas autopartistas a los requerimientos de la empresa Terminal (en este caso, Toyota), en la medida que los horarios diarios de trabajo quedan supeditados a su demanda.

Ante la necesidad de las terminales automotrices de recepcionar los productos fabricados por la empresa con mayores frecuencias semanales, y a efectos de no desabastecer a los clientes, se podrá solicitar la aplicación de sistemas que permitan trabajar durante los siete días de la semana, otorgando los correspondientes francos compensatorios... comunicándolo previamente a la representación gremial (CCT 665/04).

Por su parte, las modalidades de contratación que se expresa en los CCT están vinculadas al nivel "macro", siendo los estipulados por la Ley de Contrato de Trabajo. El conjunto de CCT analizados habilita a la firma a hacer uso de las formas promovidas que se determinasen por ley. En este mismo orden, el carácter de prueba de los trabajadores en la empresa pasó de seis meses, en la década del 90 a tres en la nueva ley, lo que se incorporó a los CCT. No obstante, en el CCT firmado en Master Trim se alude a la posibilidad 
que la firma pueda contratar personal a través de "empresas de servicios eventuales" hasta un plazo de 12 meses.

Este ejercicio que aparece como simple puesta en práctica de las posibilidades que brinda el mercado de trabajo y las regulaciones vigentes como mecanismos seleccionadores y de pautas para enfrentar fácilmente los avatares de la demanda, marca las condiciones de heterogeneidad entre los trabajadores. Pero no solo entre los trabajadores de diferentes empresas, sino al interior de ellas, se crean mecanismos que tienden a dividir al colectivo de trabajo a partir de la introducción de criterios vinculados a la flexibilidad externa, con distinciones que actúan como disciplinadores entre los temporarios y los permanentes. "Esto implica una escisión entre un "núcleo estable" de trabajadores protegidos y una "periferia” de trabajadores precarizados" (Palomino 2004:26).

En términos salariales, pueden observarse continuidades en torno a las formas de su composición. En este sentido, todos los CCT analizados incluyen, además del salario básico, una parte variable conformada por distintos indicadores dependiendo de la firma. En el caso de la terminal automotriz, la parte variable del salario está determinada por tres indicadores: productividad, calidad e implicación, en la medida que las sugerencias como la participación en equipos Kaizen comprenden un pago adicional. Por parte de la empresa Yazaki, este indicador se conforma por la calidad, comprendida por las devoluciones realizadas por las firmas terminales. Asimismo, la empresa productora de parabrisas incluye un adicional vinculado a la calidad y los objetivos de producción de la firma. En torno a Master Trim, el indicador del pago variable incluye estas dos categorías y además se incorpora una tercera que refiere al empeño individual, compuesta por el presentismo, seguridad y empeño, esta última vinculada a la disciplina del trabajador. Si uno de los rasgos distintivo que había marcado la firma de los CCT había sido la incorporación de cláusulas de individualización salarial vía pagos variables; en el actual entorno esto se sigue manteniendo en los mismos contenidos, tanto en aquellos firmados recientemente como los que se han renovado en los últimos años.

Así, se desprende que existe una cantidad de mecanismos tendientes a facilitar la heterogeneización del colectivo de trabajadores, erosionando los elementos que posibilitaban la concreción de una identidad vinculada al trabajo y a los trabajadores. En primera instancia, la heterogeneización productiva como rasgo característico de división del trabajo produce sus efectos sobre la división laboral, profundizada en los distintos espacios de trabajo por medio de los mecanismos mencionados. Es decir, no solo se procesa una división laboral vía heterogeneización productiva, sino también una fuerte heterogeneización al interior de cada una de las firmas. Al respecto, la existencia de diferentes 
tipos de trabajadores por su forma contractual juega un rol central como mecanismo de heterogeneización en un doble sentido. Por una parte, la situación misma de inserción precaria actúa como forma manifiesta de control, en la medida en que quienes están en esa condición internalizan las expectativas de las direcciones empresarias, ajustando su conducta a la demanda de la firma, por otra parte, actúa sobre los trabajadores permanentes, que se ven presionados por la existencia de aquellos y ambos se ven compelidos a aceptar los designios de la empresa en la medida en que el fantasma de la desocupación pende sobre ellos.

De esta forma, los CCT analizados permiten observar que los cambios desarrollados en sus contenidos son escasos y se mantienen las lógicas dominantes en los 90, sobre todo en aquellos aspectos vinculados a la flexibilidad interna, en tanto los aspectos vinculados a la externa, los factores "macro" (cambio en la ley de contrato de trabajo), influyen en el nivel de la empresa aunque, en este último sentido, algunos CCT permiten incorporar la disponibilidad de los trabajadores a través del personal contratado por medio de agencias de personal temporario o a plazo. A su vez, en el marco del análisis desarrollado, la división de empresas como forma de división técnica del trabajo se constata también en esos CCT, donde se pueden vislumbrar las formas que adquiere la organización del proceso de trabajo y el efecto que tiene la terminal sobre sus trabajadores y los del conjunto de empresas proveedoras. Así, la producción de la terminal impacta directamente sobre el conjunto de los trabajadores, imponiendo los ritmos a trabajadores de otras firmas, a la vez que por medio de los CCT se permite la presencia de trabajadores con contratos a plazo. Esto, como se pudo ver, introduce una ruptura también dentro de los trabajadores de una misma firma, reforzando los procesos de individualización de las relaciones laborales, es decir, división de trabajadores por empresas y división de trabajadores dentro de cada una de las firmas.

\section{CONCLUSIONES}

En este trabajo intentamos pensar cómo las lógicas de funcionamiento y los cambios desarrollados desde el Estado, a través de mecanismos de intervención y regulación, las transformaciones en materia económica y su impacto en el mercado de trabajo, tuvieron efecto en el nivel de la firma, tomando un aspecto que ha sido central durante la década de 1990, como fue la negociación colectiva y, por medio de ella, la introducción de mecanismos de flexibilidad. Este aspecto, si bien no conforma la totalidad de las variables vinculadas a las relaciones laborales en la firma, permite mirar la evolución de los crite- 
rios de flexibilidad y comparar dos periodos, que como fue dicho, presentan diferencias importantes en el nivel "macro".

En este nivel se han observado cambios importantes a partir de las sanciones de leyes que les otorgaron a los trabajadores la posibilidad de posicionarse mejor frente al capital en relación al período "neoliberal". Esto se tradujo en la recuperación de un conjunto de derechos amenazados durante la década del 90, la restitución de otros y la moderación de algunas de las lógicas de flexibilidad, como los casos del sistema de pasantías, el acortamiento del período de prueba y el incremento de las negociaciones colectivas para la determinación de mejoras salariales. Ello, junto con la recuperación del empleo, posibilitó una revitalización del actor sindical, lo que procesó un círculo virtuoso aplacando las condiciones vinculadas a las prácticas de la década anterior.

No obstante, la producción de estos elementos en el nivel "macro" parece haber tenido un efecto parcial en la negociación colectiva del sector automotriz en el nivel de la firma, más específicamente en los cambios producidos, tanto cuantitativa como cualitativamente, en el marco los CCT. En este sentido, pudo observarse que bajo el nuevo modelo siguió desarrollándose una negociación a nivel de empresa que, en términos de firma de CCT por año, fue mayor durante el actual período que en el anterior.

En términos cualitativos, estos nuevos CCT fueron incorporando cláusulas en su contenido vinculados a los cambios en la legislación, aunque también supuso la incorporación de mecanismos de prácticas de flexibilidad externa como la integración de la posibilidad de incrementar el empleo por medio de la contratación de operarios de agencias de personal temporario. Asimismo, se sostuvieron las lógicas de flexibilidad interna vinculadas a las formas de producción, la realización de tareas y el sostenimiento de polivalencia como mecanismo de baja salarial. A ello pueden sumársele otras instancias como el sostenimiento de pagos vinculados a objetivos, ya sean por productividad o calidad, y también los mecanismos de evaluación como formas de disciplinamiento.

En síntesis, si bien los cambios producidos en el nivel "macro" han sido importantes, estos se han reflejado en la firma de nuevos CCT de manera limitada, sosteniéndose en sus contenidos las lógicas de flexibilidad incorporadas en el período anterior y con ello la posibilidad de individualizar las relaciones laborales en el nivel de la firma. Asimismo, cabe destacar que el análisis llevado adelante forma parte de una de las variables a ser estudiadas en las relaciones laborales. Quedaría por verse en otros análisis la evolución del conflicto, las estrategias de los actores intervinientes, la gestión de la fuerza de trabajo y su impacto sobre las subjetividades, elementos que exceden las posibilidades de este análisis. 
Pareciera que, en tiempos prolíficos en negociación salarial y en sus aumentos, ha quedado relegado el análisis político de los efectos reales y contundentes que ha tenido la década del 90 sobre las condiciones generales del ejercicio del trabajo. Es necesario reconocer que los esfuerzos legislativos por revertir una situación global adversa para los trabajadores, no tienen un efecto inmediato sobre una nueva cultura impuesta en lo que refiere a las relaciones entre capital y trabajo en los espacios acotados de las firmas. Pero el Estado, y más precisamente la autoridad ministerial que corresponde a la esfera de las relaciones laborales, no se ha dado el trabajo de realizar una tarea seria de investigación en este sentido. Como decimos, no se puede esperar que la ley tenga impacto directo, y por su sola existencia, en la realidad de las personas, es necesario actuar en consecuencia y construir las herramientas necesarias para revertir procesos que, a pesar de los cambios en el nivel macro de las relaciones laborales, siguen actuando en el nivel micro manteniendo realidades que sostienen un entramado cultural que tiende a vulnerar la seguridad, el ambiente y la condición de los trabajadores.

\section{REFERENCIAS}

Berasueta, Ana Cecilia y Eugenio Biaffore. 2010. "Principales reformas normativas en el ámbito laboral. Período 2002/2009.” En Transformaciones del empleo en la Argentina, editado por J. C. Neffa, D. Panigo y P. Pérez. Buenos Aires: CEIL-PIETTE.

Bilbao, Andrés. 1999. "La globalización y las relaciones laborales." Cuadernos de relaciones laborales 15:123-37.

Boyer, Robert.1987. La flexibilidad laboral en Europa. Madrid: Ministerio de Trabajo y Seguridad Social.

Delfini, Marcelo. 2010. "Prácticas y estrategias empresarias para la reproducción de la dominación en los espacios de trabajo. Formas de control obrero en el sector automotriz." Trabajo y Sociedad 14 (3):1-20.

Español, Paula y Germán Herrera. 2010. "Empleo industrial en la posconvertibilidad. Una aproximación del período 2003-2008 bajo una mirada de largo plazo." En Transformaciones del empleo en la Argentina. Estructura, dinámica e instituciones, compilado por J. C. Neffa, D. Panigo y P. Pérez. Buenos Aires: Ciccus.

Figari, Claudia. 2000. "Formas de disciplinamiento y nuevas selectividades en la modernización empresaria: modalidades del control técnico y social en los 90.” Ponencia presentada en el Congreso ALAST, Buenos Aires. 
Linhart, Daniele. 2002. “Los asalariados y la mundialización.” Sociología del Trabajo 45: 53-68.

Ministerio de Trabajo, Empleo y Seguridad Social (MTESS). 2010. Convenios Colectivos de Trabajo (CCT): 190/96 E, 211/97E; 1033/09 E449/01 E, 665/04 E, 614/03 E, 98/94 E, 475/02 E, 252/04 E, 260/75, 8/89E, 234/97E, 730/05E, 475/02E, 665/04E, 704/05E, 730/05; 257/97 E; $942 / 08 \mathrm{E}$.

Montes Cató, Juan, Ana Drolas y Valentina Picchetti. 2005. "Las relaciones de poder en los espacios de trabajo." Pp. 93-120 en Estado y relaciones laborales, compilado por A. Fernández. Buenos Aires: Prometeo.

Montes Cató, Juan, Paula Lenguita y Paula Varela. 2010. “Trabajo y política en Argentina: la potencialidad de la acción gremial en el lugar de trabajo.” Estudios Políticos 1(2):1-19.

Novick, Martha. 1998. "Reconversión segmentada en la Argentina: empresas, mercado de trabajo y relaciones laborales a fines de los 90.” Pp. 51-72 en Reestructuración productiva, mercado de trabajo y sindicatos en América Latina, compilado por E. De la Garza. Montevideo: OITCentro Interamericano de Investigación y Documentación sobre Formación Profesional.

Observatorio del Derecho Social, Central de Trabajadores de la Argentina. 2010. Conflictividad laboral y negociación colectiva. Informe anual 2009. Buenos Aires: Central de Trabajadores de la Argentina.

Palomino, Héctor. 2004. “Trabajo y teoría social: conceptos clásicos y tendencias contemporáneas." Pp. 28-38 en Multiculturalismo y democracia. Bolivia, editado por H. Palomino, J. E. Vega, J. M. Iñiguíz. Bolivia: PNUD.

Palomino, Héctor y David Trajtemberg. 2007. "Nueva dinámica de las relaciones laborales y la negociación colectiva en Argentina.” Revista Trabajo MTEySS 3:47-70.

Senén González, Cecilia y Héctor Palomino. 2006. "Diseño legal y desempeño real: Argentina." Pp. 95-166 en Diseño legal y desempeño real: instituciones laborales en América Latina, editado por G. Bensusán. México: UAM. 